\title{
Estudo hipotético para avaliação preliminar da resiliência na mobilidade urbana
}

\author{
Hypothetical study for a preliminary evaluation of \\ resilience in urban mobility
}

\section{Marcel Carlos Martins Antônio Nélson Rodrigues da Silva}

\section{Resumo}

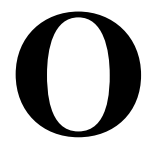

objetivo deste estudo é desenvolver uma estratégia de avaliação da resiliência da mobilidade urbana. A abordagem é baseada em um cenário hipotético em que transportes motorizados estariam impossibilitados de ocorrer por restrições diversas. Portanto, apenas os modos a pé e bicicleta foram considerados para este exercício teórico. As viagens foram inicialmente classificadas em dois grupos, de acordo com sua adaptabilidade ou transformabilidade, sendo as do primeiro grupo consideradas resilientes. Uma terceira categoria teve que ser introduzida, no entanto, para representar outro conjunto de viagens resilientes. Estas são as viagens excepcionais, isto é, viagens a pé ou de bicicleta que são mais longas do que as Distâncias Máximas Possíveis (DMP) definidas para a avaliação da resiliência. Em uma aplicação do método realizado na cidade de São Carlos, SP, com DMP de 3 $\mathrm{km}$ e $8 \mathrm{~km}$ para viagens a pé e de bicicleta respectivamente, 97,7\% das viagens foram classificadas como resilientes, o que é um resultado positivo.

Palavras-chave: Planejamento de transportes. Modos ativos. Resiliência. Mobilidade urbana.

${ }^{1}$ Marcel Carlos Martins ${ }^{1}$ Universidade de São Paulo São Carlos - SP - Brasil

${ }^{2}$ Antônio Nélson Rodrigues da Silva

${ }^{2}$ Universidade de São Paulo São Carlos - SP - Brasil

Recebido em 09/05/17 Aceito em 23/04/18

\section{Abstract}

The aim of this study aims is to develop a strategy to evaluate the resilience of urban mobility. The approach is based on the assumption that no forms of motorised transport would be available. Hence, only walking and cycling were considered as urban transport modes for the purpose of this theoretical exercise. The trips were initially classified in two groups, according to their adaptability or transformability. Those in the first group are considered resilient trips. However, a third category had to be created to represent another set of resilient trips. These are the exceptional trips, i.e. walking or cycling trips that are longer than the Maximum Possible Distances (MPD) defined for the evaluation of resilience. In an application of the method conducted in the city of São Carlos - SP, with MPDs of 3 $\mathrm{km}$ (for walking) and $8 \mathrm{~km}$ (for cycling), $97.7 \%$ of the trips were classified as resilient, which is a positive outcome.

Keywords: Urban transportation. Active modes. Resilience. Urban mobility. 


\section{Introdução}

Não há novidade alguma na afirmação de que os combustíveis fósseis são recursos energéticos limitados. Seu papel no setor de transportes é crucial (FERNANDES et al., 2015) e cresce continuamente, especialmente em países ao sul do globo (SCHWANEN, 2016). Porém, o chamado "pico do petróleo" pode trazer discrepâncias na oferta e demanda dos combustíveis a um ponto sem retorno (HUBBERT, 1949). A falta de investimentos em modos ativos de transportes e em tecnologias de substituição aos combustíveis fósseis torna as condições ainda mais preocupantes.

Não somente o setor de transporte, tanto público como privado, se mostra vulnerável, como também as próprias cidades se tornam dependentes de veículos motorizados. O espraiamento urbano impede que origens e destinos estejam próximos o suficiente para serem percorridos por modos ativos. Em cidades espraiadas, alguns autores afirmam que o consumo de energia é muito maior; consequentemente, a demanda por combustíveis fósseis também (NEWMAN; KENWORTHY, 2011; SAUNDERS; RODRIGUES DA SILVA, 2009; RODRIGUES DA SILVA; COSTA; BRONDINO, 2007). As distâncias cada vez maiores, assim como outros fatores, desencorajam os modos a pé e de bicicleta em viagens diárias. Essas influências apenas se somam à demanda por veículos motorizados, o que aumenta a vulnerabilidade das cidades diante de algum evento restritivo. Nesse sentido, a resiliência na mobilidade está relacionada à capacidade do transporte urbano de resistir e de se adaptar. Mencionado isso, este trabalho tem como objetivo elaborar uma metodologia de avaliação da resiliência na mobilidade urbana. Ainda que esse termo seja amplo e seu entendimento complexo, o trabalho visa, de forma preliminar, mostrar a dependência aos veículos motorizados nas regiões urbanas. Uma restrição sobre veículos motorizados significa que as viagens podem apenas ser realizadas pelo modo a pé ou de bicicleta.

\section{Referencial teórico}

Nesta seção são abordados quatro aspectos:

(a) a demanda crescente de combustíveis fósseis como fonte de energia, especialmente no setor de transportes;

(b) o fenômeno do espraiamento urbano como causa e consequência do aumento no uso de veículos motorizados nas viagens diárias; (c) a substituição de modos ativos para curtas distâncias por veículos motorizados e outros fatores de influência; e

(d) a apropriação do termo "resiliência na mobilidade”, usado por Fernandes et al. (2015), na análise realizada nesta pesquisa.

Uma revisão de trabalhos anteriores relacionados ao tema também é feita ao final deste item.

É de se imaginar a importância dos combustíveis fósseis no fornecimento de energia global. No setor de transportes não é diferente. O petróleo é responsável por $57 \%$ da energia consumida nesse setor, e o gás natural, por 3\% (FERNANDES et al., 2015). Apesar de alguns estudos apontarem para uma redução do uso do carro em alguns países desenvolvidos (NEWMAN; KENWORTHY, 2011), a demanda por transporte motorizado nos países em desenvolvimento continua em forte crescimento (SCHWANEN, 2016). Por ser um produto limitado, o petróleo e seu crescente uso como fonte de energia causam preocupação. Dantas, Page e Krumdieck (2010) referem-se ao chamado "pico do petróleo" como o ano em que a produção mundial de petróleo atinge seu ápice e, em seguida, decai. Essa futura discrepância entre demanda e oferta, apontada primeiramente por Hubbert (1949), pode atingir de várias formas o setor de transportes. Apesar de existirem medidas para reduzir essa dependência, a liberdade pessoal e a flexibilidade do veículo individual, somadas ao retorno lucrativo dos investimentos na indústria do petróleo, apenas apontam para um futuro cada vez mais dependente dos veículos movidos a combustível fóssil.

Outro fator que incentiva o uso dos veículos motorizados é o espraiamento urbano. Segundo Brunner (2013), esse é um fenômeno cuja expansão para além dos limites urbanos causa altos níveis de segregação e dependência do carro. Brueckner (2000) acredita que o aumento populacional, o aumento da renda e a redução nos custos do transporte foram forças que resultaram no espraiamento. Essa expansão desorganizada acaba por aumentar as distâncias de viagens diárias; logo, aumenta-se a necessidade por transporte motorizado. Lovelace e Philips (2014), em seu estudo nas regiões de Yorkshire e Humber, descobriram que um terço das viagens diárias ultrapassava os $10 \mathrm{~km}$, ao mesmo tempo em que consumia três quartos da energia total da região. Para Brunner (2013), o espraiamento é um fenômeno complicado, cuja eliminação não é tarefa fácil. Todavia, estudos voltados para achar soluções são muitos. Alguns autores (SHIM et al., 2006; 
DANTAS; PAGE; KRUMDIECK, 2010; BUEHLER et al., 2011; HAMIDI; EWING, 2015) apresentam estratégias de combate ao espraiamento e seu alto consumo energético. Ao mesmo tempo, políticas de descentralização e uso misto do solo aparecem como soluções viáveis (BUEHLER et al., 2011; ZHANG; KOCKELMAN, 2014).

O fenômeno do espraiamento, juntamente com o crescente uso do transporte motorizado, vem levando à diminuição do uso de modos ativos em viagens de curta distância. Hydén, Nilsson e Risser (1999) mostraram que a média de viagens a pé na Europa não passa de 1 viagem por pessoa/dia, com maioria limitada a $1 \mathrm{~km}$. As viagens de bicicleta seriam em média 2,5 por pessoa/dia, limitada a 5 $\mathrm{km}$. Outros estudos também demonstraram a limitação do modo a pé em torno de $1 \mathrm{~km}$, e da bicicleta, em até $3 \mathrm{~km}$ (YANG; DIEZ-ROUX, 2012; LARSEN; EL-GENEIDY; YASMIN, 2010; OLIVEIRA; TAN; RODRIGUES DA SILVA, 2016). Entretanto, alguns autores se utilizam de valores maiores em seus estudos. Dantas, Page e Krumdieck (2010), em sua avaliação de risco ao transporte para o caso de diminuição na oferta de combustível, adotam $2 \mathrm{~km}$ para o modo a pé e $15 \mathrm{~km}$ para o modo bicicleta. Philips (2014), por sua vez, assume que qualquer pessoa pode percorrer 8 km de bicicleta diariamente com base no que é recomendado pelo governo britânico.

Claramente, é possível o ser humano percorrer distâncias maiores que as mencionadas, seja a pé ou de bicicleta. Entretanto, fora a própria existência do veículo motorizado, usado até mesmo em curtas distâncias, existem outros fatores que podem desincentivar modos mais ativos. Philips (2014) apontou, entre outros, o tempo de viagem, o relevo e o clima como fatores de influência na escolha do modo. Já Hydén, Nilsson e Risser (1999) listaram seis fatores de influência: valor social (do veículo), saúde, estética, conforto, mobilidade e segurança. Outros estudos também mostraram a idade, a percepção de segurança, a estética, o relevo e a distância entre origem e destino como cruciais para a decisão do modo de transporte (BOHANNON, 1997; MOUDON et al., 2006; HORNING; ELGENEIDY; KRIZEK, 2007; MCCORMACK et al., 2008; FERNANDES et al., 2015).

É com base nessas condições de possível escassez/diminuição da oferta dos combustíveis fósseis e dependência do veículo motorizado que o conceito de resiliência é inserido. Entre as várias definições encontradas na literatura (FOLKE et al., 2010; WALKER; COOPER, 2011; SCHWANEN, 2016), é possível entender a resiliência como uma capacidade adaptativa diante de uma situação que põe em risco um sistema. Tratando-se da resiliência relacionada a crises de combustível, alguns trabalhos merecem menção. Em sua análise com indicadores econômicos, sociais e energéticos, Exner et al. (2016) descobrem que a resiliência dos municípios da Áustria é moderada. Regiões com maior atividade rural se mostraram mais resilientes, uma vez que conseguem manter uma conexão para a distribuição de alimentos para toda a comunidade.

Um foco maior é dado à mobilidade por Dantas, Page e Krumdieck (2010), que, apesar de não mencionarem diretamente o termo "resiliência", avaliam o risco ao transporte e planejamento urbano para o caso da diminuição da oferta de combustível. Eles categorizam as viagens impactadas por sua essencialidade, como visto a seguir:

(a) opcionais: viagens que podem ser eliminadas sem perda de bem-estar;

(b) necessárias: viagens que, uma vez eliminadas, causam perda social e oportunidades econômicas; e

(c) essenciais: viagens que pessoas teriam dificuldade em eliminar, pois causaria danos à saúde e à economia, além de limitar o acesso a necessidades básicas.

Eles fazem o mesmo com o nível de impacto, que é dividido da seguinte forma:

(a) baixo impacto: a disparidade de fornecimento ocorreria, porém sem a necessidade de restrições de viagens;

(b) médio impacto: viagens opcionais são eliminadas para sustentar a disparidade entre oferta e demanda;

(c) alto impacto: viagens necessárias são eliminadas; $\mathrm{e}$

(d) impacto muito alto: viagens essenciais são eliminadas.

Os resultados estimados para a região metropolitana de Christchurch, Nova Zelândia, para o ano de 2041 mostraram que a região com estrutura concentrada teria possibilidade de maiores mudanças do modo automóvel para modo a pé e de bicicleta. A região de Christchurch também foi foco da pesquisa de Rendall et al. (2011), que introduziram o conceito de Active Mode Accessibility (AMA), ou seja, a proporção de atividades que podem ser realizadas por modos ativos. Um AMA alto, por exemplo, significa menor necessidade energética para alimentar o sistema, logo maior resiliência para responder a crises de combustível e outras restrições. Junto com os modos a pé e de bicicleta, porém, os autores também usaram o transporte público na soma dos modos ativos. Seus resultados mostraram que as atividades na região central de Christchurch poderiam ser completamente realizadas por qualquer um dos três modos. 
Por sua vez, Philips (2014) se utiliza de microssimulação para estimar o potencial dos modos a pé e de bicicleta diante de crises de combustíveis na Inglaterra. Adotando $8 \mathrm{~km}$ como a distância máxima para os modos ativos, seus resultados indicaram que apenas $44 \%$ dos indivíduos de sua amostra nacional teriam capacidade de se deslocar para o trabalho caminhando ou de bicicleta. Para descrever a resiliência do transporte diante de tais crises, Philips estabelece como mais apropriado o termo de “resiliência evolucionária”.

Ao realizar a pesquisa por trabalhos relacionados à resiliência afetando diretamente 0 transporte urbano, percebeu-se que o material ainda é mínimo, especialmente se tratando da escassez de combustível. Além disso, os trabalhos encontrados são focados em regiões de países desenvolvidos, onde o processo de expansão urbana se deu de forma diferenciada se comparado com o de países em desenvolvimento como o Brasil, por exemplo.

Em vista disso, visando acrescentar ao debate da resiliência a combustíveis fósseis, neste trabalho é realizada a análise de uma cidade em um país em desenvolvimento através da elaboração de uma metodologia de avaliação. Para isso, adota-se o termo "resiliência na mobilidade" usado por Fernandes et al. (2015) como a capacidade de persistência e adaptabilidade diante do risco à mobilidade urbana. De forma mais específica, analisa-se a resiliência ao ser restringido o uso de veículos motorizados. A segmentação dessa resiliência também segue o proposto por Folke et al. (2010): em persistência, adaptabilidade e transformabilidade. A persistência está relacionada com a continuidade do modo usado, sem comprometer o bem-estar e a qualidade de vida. Já a adaptabilidade equivale à mudança de modo visando a não comprometer o bem-estar do indivíduo ou do grupo. Por fim, a transformabilidade se encaixa onde a persistência e a adaptabilidade não são possíveis, e onde são necessárias novas condições para se enquadrar nos dois primeiros segmentos.

\section{Método}

Nesta seção é apresentada a estratégia proposta para a avaliação da resiliência na mobilidade em uma cidade. No primeiro tópico é definido o que são as
Distâncias Máximas Possíveis (DMP). O segundo tópico aborda a segmentação da resiliência para as viagens a partir do trabalho de Folke et al. (2010). Os tipos de viagem e os níveis de impacto usados neste trabalho foram baseados no estudo de Dantas, Page e Krumdieck (2010). Uma vez que se optou por trabalhar com impactos considerados altos e muito altos, as viagens englobadas são consequentemente as necessárias e as essenciais.

\section{Distâncias máximas possíveis}

A metodologia parte do pressuposto de que certas distâncias podem ser percorridas pelo indivíduo sem a necessidade de veículo motorizado, ou seja, apenas com o uso de bicicleta ou a pé. É importante lembrar que essas distâncias, aqui chamadas de Distâncias Máximas Possíveis (DMPs), devem ser maiores que as distâncias aceitáveis ou desejáveis para o indivíduo percorrer a pé ou de bicicleta, pois em uma restrição como a que é apresentada o uso de transporte motorizado está impossibilitado, e não há alternativa senão andar ou pedalar.

Após estabelecer valores de $\mathrm{X}$ e $\mathrm{Y}$ como sendo as DMPs a pé e de bicicleta respectivamente, e definir os tipos de viagens a serem analisadas, devem ser observadas as zonas dentro da matriz de origem e destino da região em avaliação. Com a ajuda de ferramentas de um sistema de informações geográficas (SIG), são registradas as distâncias de uma zona à outra. Essas distâncias são medidas entre pontos previamente estabelecidos como centroides ou centros aproximados das zonas, que, caso não sejam indicados pela ferramenta usada, podem ser adotados pelo próprio avaliador (Figura 1).

Uma vez encontradas as distâncias entre as zonas, é possível compará-las com os valores de X e Y das DMPs. As condições, então, são as seguintes:

(a) se $d \leq X$, o trajeto entre as regiões pode ser feito andando ou de bicicleta;

(b) se $\mathrm{X}<\mathrm{d} \leq \mathrm{Y}$, a distância é demasiada longa para o modo a pé, porém pode ser percorrida de bicicleta; e

(c) se d > Y, as zonas são muito distantes para serem percorridas diariamente sem o uso de transporte motorizado, e em caso de restrição o indivíduo será necessariamente afetado. 
Figura 1 - Distância entre centroides de zonas hipotéticas

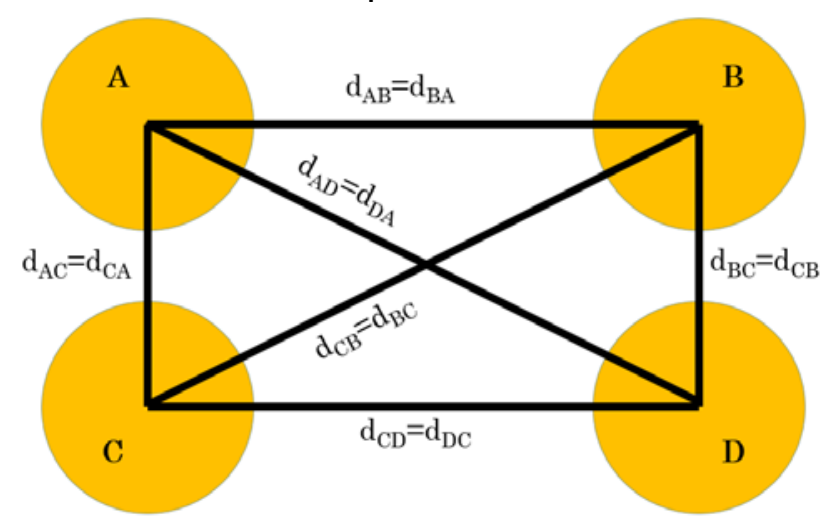

As viagens realizadas dentro de uma mesma zona serão consideradas iguais a zero $\left(\mathrm{d}_{\mathrm{AA}}=\mathrm{d}_{\mathrm{BB}}=\mathrm{d}_{\mathrm{CC}}=\right.$ $\mathrm{d}_{\mathrm{DD}}=0$ ); assim, podem ser realizadas a pé ou de bicicleta. Quanto às viagens entre diferentes zonas, terão o mesmo valor, independentemente do sentido. Por exemplo, a viagem de A a B terá a mesma distância da viagem de $\mathrm{B}$ a $\mathrm{A}\left(\mathrm{d}_{\mathrm{AB}}=\mathrm{d}_{\mathrm{BA}}\right)$, e assim por diante.

\section{Segmentação da resiliência}

Com as DMPs estabelecidas e as distâncias entre zonas em mãos, é elaborada então a matriz de modo adequado de viagem, em que são fixados os trajetos possíveis de ser percorridos a pé ou de bicicleta, somente de bicicleta, ou somente por veículo motorizado. Em seguida, os modos usados na matriz de origem e destino são comparados com os da matriz de modo adequado. Com base na divisão de partes apresentada por Folke et al. (2010), é estabelecido o número de viagens que se caracterizam como de persistência, de adaptabilidade e de transformabilidade.

As DMPs são escolhidas de forma que o máximo de pessoas, independentemente da idade ou do relevo do terreno, por exemplo, possam percorrê-las a pé ou de bicicleta. Com isso, é esperado que algumas pessoas, em melhor forma física, ou em locais de relevo menos acidentado, percorram distâncias maiores que as estabelecidas sem maiores problemas. A essas viagens feitas a pé ou de bicicleta com distâncias maiores que as DMPs foi dado o nome de viagens excepcionais. Esse novo tipo de viagem é somado às viagens permanentes $\mathrm{e}$ adaptáveis, e consideradas resilientes. O nível de resiliência aqui considerado pode ser obtido através da escala da Tabela 1. As viagens transformáveis, por outro lado, serão enquadradas como vulneráveis. Sua escala de vulnerabilidade será inversamente proporcional à de resiliência.

\section{Resultados}

Um exemplo de aplicação da metodologia proposta foi feito na cidade de São Carlos, SP. O banco de dados utilizado foi o da pesquisa de origem e destino e dos diários de viagens, realizados nos anos de 2007-2008, com um total de 15.885 viagens. Embora os dados sejam relativamente antigos, o objetivo do trabalho foi apresentar a proposta metodológica para a avaliação da resiliência; logo, entendeu-se que para isso não haveria necessidade de atualização dos dados. O levantamento foi feito durante todos os horários do dia, contabilizando tanto as viagens para o trabalho, por exemplo, como o retorno para casa. Viagens com destino não informado ou com modo de transporte que não fosse motorizado, a pé ou de bicicleta, foram excluídas da pesquisa. Todas as viagens cujo modo era motorizado foram unidas em uma única descrição, veículo motorizado, somando um total de 8.410 viagens. As viagens a pé e de bicicleta foram categorizadas individualmente, totalizando 4.576 e 448 viagens respectivamente. Quanto ao motivo da viagem, foram mantidos apenas os considerados necessários ou essenciais, conforme Dantas, Page e Krumdieck (2010). As viagens cujo motivo não foi informado ou aquelas consideradas opcionais (lazer) foram excluídas. No fim, o número total de viagens usadas na pesquisa foi de 13.425.

Para estabelecer as distâncias entre as zonas foi usada a matriz de distâncias entre as 41 zonas de tráfego já definidas dentro da área urbana do município (Figura 2). As distâncias entre os centroides das zonas de tráfego foram medidas através da malha viária urbana. Viagens intrazonais foram consideradas com distância zero, mesmo para zonas com grandes áreas (ex.: 10 e 31), pois nestes casos grande parte de sua população se concentra em áreas menores dentro das próprias zonas. As maiores distâncias encontradas foram listadas na Tabela 2. 


\section{Adoção das distâncias máximas possíveis}

Para a adoção das DMPs foi preciso levar em conta que essas distâncias variam conforme o indivíduo e a região. Pessoas de mais idade percorrem menores distâncias que pessoas mais novas, assim como condições de saúde e físicas limitam o uso de modos ativos. Cidades mais planas tendem a ser mais fáceis de serem percorridas a pé e de bicicleta do que cidades com relevo muito acidentado, entre outros fatores.

Tabela 1 - Nível de resiliência na mobilidade

\begin{tabular}{c|c|c|c|c|c}
\hline $\begin{array}{c}\text { Nível de } \\
\text { resiliência }\end{array}$ & $\begin{array}{c}\text { Muito } \\
\text { baixa }\end{array}$ & Baixa & Média & Alta & Muito alta \\
\hline $\begin{array}{c}\text { Viagens } \\
\text { resilientes (\%) }\end{array}$ & $0-20,0$ & $20,1-40,0$ & $40,1-60,0$ & $60,1-80,0$ & $80,1-100$ \\
\hline
\end{tabular}

Figura 2 - Zonas de tráfego na cidade de São Carlos

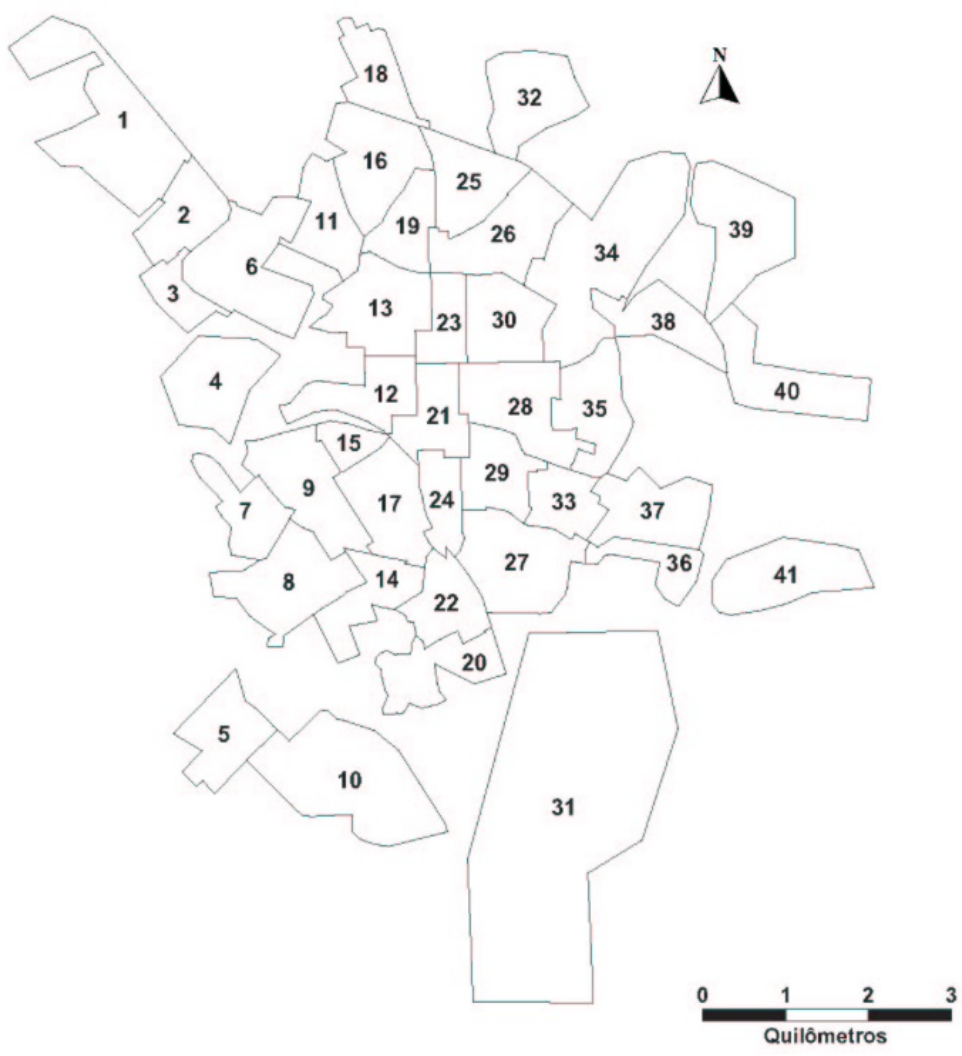

Tabela 2 - Maiores distâncias entre centroides das zonas

\begin{tabular}{c|c|c}
\hline Distâncias (km) & \multicolumn{2}{|c}{ Zonas de O\D } \\
\hline 12,97 & 5 & 40 \\
12,27 & 12 & 40 \\
11,73 & 1 & 41 \\
11,66 & 1 & 31 \\
11,66 & 1 & 40 \\
11,60 & 1 & 10 \\
11,24 & 5 & 39 \\
10,75 & 5 & 32 \\
10,55 & 10 & 39 \\
10,34 & 8 & 40 \\
\hline
\end{tabular}


Mencionado isso, e com base na revisão bibliográfica, os valores adotados como DMPs foram de $3 \mathrm{~km}$ para as viagens a pé e de $8 \mathrm{~km}$ para as viagens de bicicleta. É importante observar que é possível que algumas pessoas realizem viagens com maior distância que as estabelecidas, assim como outras podem não ter condições de percorrer as DMPs. Esses valores foram estabelecidos não como máximo possível para as melhores condições, mas como uma média máxima, que visa incorporar o maior número de pessoas dentro desse intervalo.

\section{Segmentação das viagens por nível de resiliência}

Antes de analisar a segmentação da resiliência na mobilidade obtida, é interessante observar a distribuição dos modos pelas DMPs adotadas, como é visto na Tabela 3. Nela é possível perceber que existe grande quantidade de viagens feitas a pé e por veículo motorizado, mas poucas por bicicleta. Além disso, apenas 2,4\% do total de viagens ultrapassam os $8 \mathrm{~km}$. Esse dado demonstra o potencial no uso de modos ativos na cidade.

A segmentação das viagens pode ser vista na Tabela 4. Viagens classificadas como persistentes são aquelas feitas a pé ou de bicicleta. Uma vez que as distâncias são menores ou iguais às DMPs, não haveria mudança alguma após a restrição concebida. As viagens adaptáveis englobam aquelas cuja distância poderia ser percorrida a pé ou de bicicleta, porém eram feitas por veículos motorizados, e com o impedimento ao uso destes foi possível a mudança de modo sem maiores prejuízos. As viagens transformáveis são aquelas cuja distância é demasiado longa para os modos a pé e de bicicleta; assim, em caso de restrição, elas seriam afetadas. Por fim, as viagens excepcionais são as realizadas a pé ou de bicicleta, porém cobrindo distâncias maiores que as DMPs. Com $97,7 \%$ de viagens resilientes, a cidade pode ser considerada de resiliência muito alta.

Um total de 4.555 viagens (33,9\%) foi categorizado como persistente, significando que pouco mais de um terço do total de viagens se manteria. Nesse caso, as viagens intrazonais foram a maioria, como pode ser visto na Tabela 5.

As viagens adaptáveis englobaram 60,3\% do total, o que mostra que existe grande quantidade de viagens por modo motorizado que poderiam ser substituídas por modos sustentáveis, uma vez imposta uma restrição. Estas tiveram um padrão radial, com várias delas tendo a zona central 21 como origem ou destino, como é visto na Figura 3.

As 304 viagens transformáveis também apresentaram um padrão. Suas zonas de origem e destino se localizam em extremos opostos na área urbana (Figura 4) e suas distâncias ultrapassavam os $8 \mathrm{~km}$ entre os centroides.

As 469 viagens consideradas excepcionais não apresentaram nenhum padrão claro em sua distribuição. Todavia, as principais viagens nesse segmento são realizadas a pé, ultrapassando os $3 \mathrm{~km}$ estipulados como DMP. Essa quantidade demonstra que é possível distâncias maiores que as estipuladas serem percorridas por modos sustentáveis.

Tabela 3 - Distribuição das viagens por modo e por DMPs adotadas

\begin{tabular}{|c|c|c|c|c|c}
\hline Distância/Modo & A pé & Bicicleta & $\begin{array}{c}\text { Veículo } \\
\text { motorizado }\end{array}$ & $\begin{array}{c}\text { Total de } \\
\text { viagens }\end{array}$ & $\begin{array}{c}\text { Proporção } \\
\text { de viagens } \\
\text { (\%) }\end{array}$ \\
\hline De 0 a 3 km & 4.121 & 225 & 3.562 & 7.908 & 58,91 \\
De 3 a 8 km & 433 & 209 & 4.535 & 5.177 & 38,56 \\
Acima de 8 km & 22 & 14 & 304 & 340 & 2,53 \\
\hline $\begin{array}{c}\text { Total de viagens } \\
\text { Proporção de } \\
\text { viagens (\%) }\end{array}$ & 4.576 & 448 & 8.401 & 13.425 & 100 \\
\hline
\end{tabular}


Tabela 4 - Quantidade de viagens de acordo com o segmento da resiliência

\begin{tabular}{|c|c|c|c|c|}
\hline $\begin{array}{c}\text { Segmento da } \\
\text { resiliência }\end{array}$ & $\begin{array}{c}\mathrm{N}^{0} \text { de } \\
\text { viagens }\end{array}$ & $\begin{array}{c}\text { Proporção de } \\
\text { viagens (\%) }\end{array}$ & & \\
\hline Excepcional & 469 & 3,5 & \multirow{3}{*}{$97,7 \%$} & \multirow{3}{*}{$\begin{array}{c}\text { Quantidade de } \\
\text { viagens resilientes }\end{array}$} \\
\hline Persistente & 4.555 & 33,9 & & \\
\hline Adaptável & 8.097 & 60,3 & & \\
\hline Transformável & 304 & 2,3 & $2,3 \%$ & $\begin{array}{c}\text { Quantidade de } \\
\text { viagens vulneráveis }\end{array}$ \\
\hline Total & 13.425 & 100 & & \\
\hline
\end{tabular}

Tabela 5 - Principais viagens persistentes

\begin{tabular}{|c|c|c|}
\hline Número de viagens & Zona de origem & Zona de destino \\
\hline $\mathbf{6 3 4}$ & 10 & 10 \\
$\mathbf{2 2 6}$ & 8 & 8 \\
$\mathbf{1 1 9}$ & 9 & 9 \\
$\mathbf{1 0 2}$ & 1 & 1 \\
$\mathbf{8 9}$ & 18 & 18 \\
$\mathbf{7 9}$ & 26 & 26 \\
$\mathbf{5 8}$ & 2 & 2 \\
$\mathbf{5 6}$ & 29 & 29 \\
$\mathbf{5 5}$ & 34 & 34 \\
$\mathbf{5 3}$ & 17 & 17 \\
$\mathbf{5 0}$ & 26 & 34 \\
$\mathbf{4 9}$ & 34 & 26 \\
$\mathbf{4 7}$ & 20 & 22 \\
$\mathbf{4 6}$ & 22 & 20 \\
$\mathbf{4 6}$ & 6 & 6 \\
\hline $\mathbf{4 1}$ & 8 & 14 \\
$\mathbf{4 1}$ & 9 & 8 \\
\hline $\mathbf{4 0}$ & 37 & 37 \\
$\mathbf{4 0}$ & 27 & 27 \\
$\mathbf{3 9}$ & 8 & 14 \\
\hline
\end{tabular}

Figura 3 - Principais viagens adaptáveis - a espessura das linhas refere-se ao número de viagens

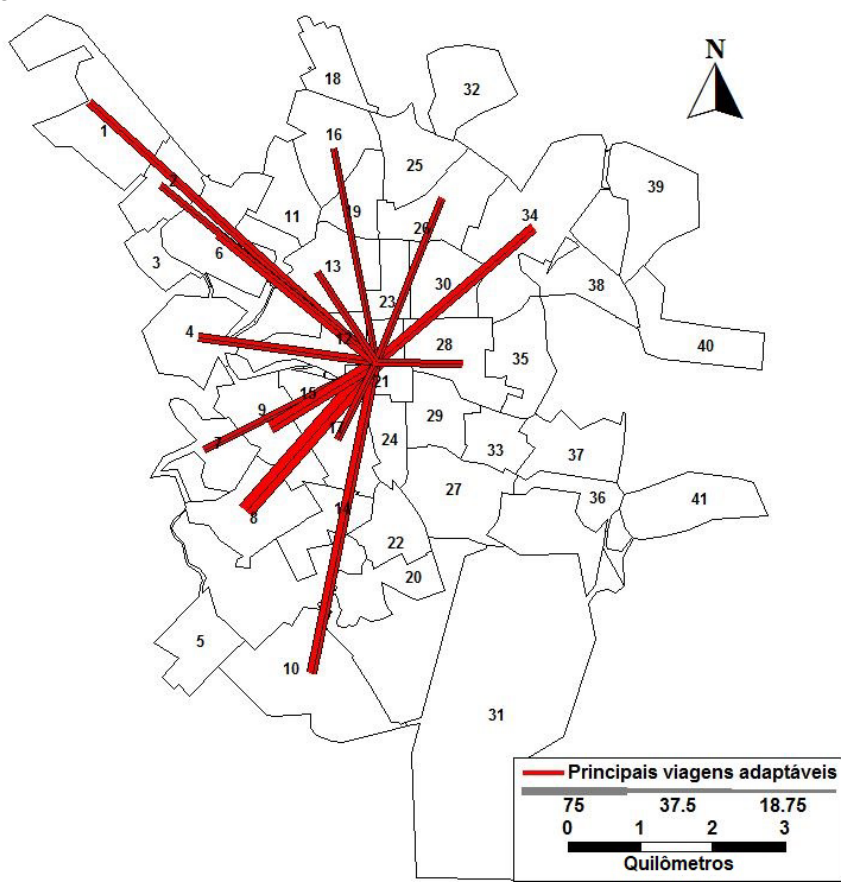


Figura 4 - Principais viagens transformáveis - a espessura das linhas refere-se ao número de viagens

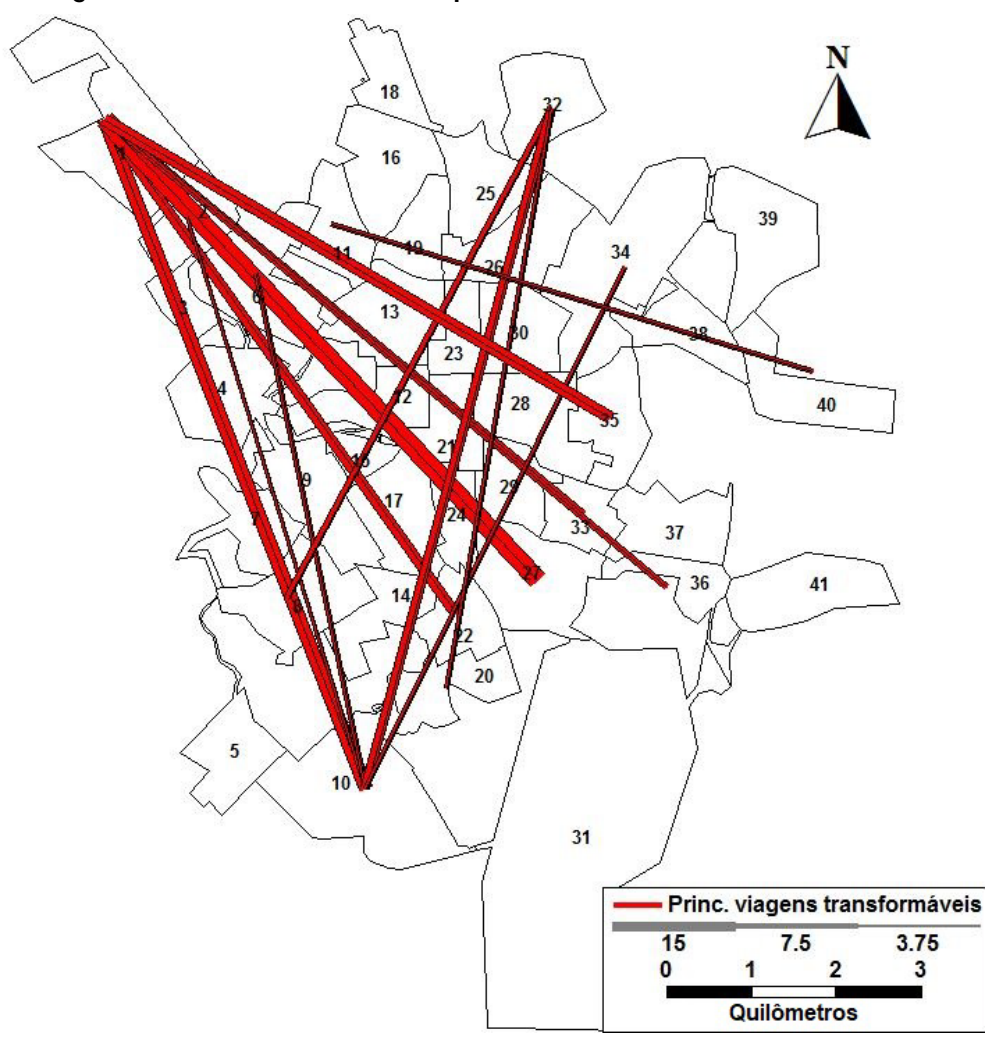

\section{Conclusão}

Este trabalho visou ao desenvolvimento de uma estratégia para avaliar a resiliência na mobilidade urbana, com sua aplicação na cidade de São Carlos, SP. No caso, foi analisada a resiliência a partir da restrição de veículos motorizados. Apenas os modos a pé e de bicicleta poderiam ser utilizados. A pesquisa trabalhou com as viagens de alto impacto e impacto muito alto, respectivamente as consideradas necessárias e essenciais de acordo com Dantas, Page e Krumdieck (2010). Da pesquisa de origem e destino, as distâncias entre os centroides das zonas foram medidas e comparadas com as distâncias máximas possíveis (DMPs) para o modo a pé e para o modo de bicicleta.

As viagens foram divididas de acordo com os estudos de Folke et al. (2010) em persistentes e adaptáveis (resilientes) e transformáveis (vulneráveis). Foi criada também uma nova segmentação, a viagem excepcional, que enquadra viagens a pé ou de bicicleta que ultrapassam as DMPs. As viagens excepcionais também são consideradas resilientes.

Os valores de resiliência na mobilidade irão variar a depender das DMPs adotadas. Estas também devem assumir valores maiores do que os estabelecidos como distâncias que o indivíduo considera aceitáveis de serem percorridas, visto que as pessoas podem caminhar distâncias maiores do se acredita que elas percorram. Para este trabalho foram adotados $3 \mathrm{~km}$ como limite para viagens a pé e $8 \mathrm{~km}$ para viagens de bicicleta.

A distribuição das viagens por modo e por DMP mostrou que $97,5 \%$ das viagens percorrem distâncias abaixo dos 8 km, limite estipulado para o uso da bicicleta. Isso demonstra o potencial de modos ativos no transporte da cidade. Os resultados também mostraram um padrão de resiliência causado pela forma da cidade: viagens pontuais (intrazonais) como persistentes, viagens radiais como adaptáveis, e viagens diametrais, transformáveis.

A cidade foi considerada de resiliência muito alta, com $97,7 \%$ das viagens (persistentes, adaptáveis e excepcionais) continuando a ser feitas mesmo considerando uma restrição a veículos motorizados. No máximo, algumas delas necessitariam de mudança de modo, porém sem maiores prejuízos ao indivíduo. Consequentemente, a vulnerabilidade foi baixa, com apenas 2,3\% das viagens caracterizadas como transformáveis. $\mathrm{O}$ número de viagens adaptáveis maior que as persistentes também indica que a maioria da população resiliente poderia migrar para modos mais sustentáveis, como já foi observado na distribuição das viagens por modo e por DMP. 
A grande quantidade de viagens adaptáveis levanta a questão do porquê estas não são feitas por modos ativos. Como observado no Referencial teórico, existem fatores que podem influenciar na tomada de decisão do indivíduo quanto ao modo de transporte. Por isso, recomenda-se para estudos futuros, a fim de se aperfeiçoar a metodologia, a análise desses fatores e também seu grau de influência na escolha do modo e nas distâncias máximas percorridas para modos ativos, sobretudo para o caso das cidades brasileiras.

\section{Referências}

BOHANNON, R. W. Comfortable and Maximum Walking Speed of Adults Aged 20-79 Years: reference values and determinants. Age and Ageing, v. 26, p. 15-19, 1997.

BRUECKNER, J. K. Urban Sprawl: diagnosis and remedies. International Regional Science Review, v. 23, n. 2, p. 160-171, 2000.

BRUNNER, A. The Effects of Urban Sprawl on Daily Life: smart growth implementation of Atlantic Stations. In: TRANSPORTATION RESEARCH BOARD ANNUAL MEETING, 92., Washington, 2013. Proceedings... Washington, 2013.

BUEHLER, R. et al. Active Travel in Germany and the U.S. Contributions of Daily Walking and Cycling to Physical Activity. American Journal of Preventive Medicine, v. 41, n. 3, p. 241-250, 2011.

DANTAS, A.; PAGE, S.; KRUMDIECK, S. Urban Form and Long-Term Fuel Supply Decline: a method to investigate the peak oil risks to essential activities. Transportation Research, v. 44, p. 306-322, 2010.

EXNER, A. et al. Measuring Regional Resilience Towards Fossil Fuel Supply Constraints: adaptability and vulnerability in socio-ecological transformations: the case of Austria. Energy Police, v. 91, p. 128-137, 2016.

FERNANDES, V. A. et al. Resiliência da Mobilidade Urbana: uma proposta conceitual. In: CONGRESSO NACIONAL DE PESQUISA EM TRANSPORTE DA ANPET, 29., Ouro Preto, Brasil, 2015. Anais... Ouro Preto, 2015.

FOLKE, C. et al. Resilience Thinking: integrating resilience, adaptability and transformability.

Ecology \& Society, v. 15, n. 4, 2010.
HAMIDI, S.; EWING, R. Is Sprawl Affordable for Americans? Exploring the association between sprawl and housing + transportation affordability. In: TRANSPORTATION RESEARCH BOARD ANNUAL MEETING, 94., Washington, 2015. Proceedings... Washington, 2015.

HORNING, J.; EL-GENEIDY, A.; KRIZEK, K. J. Perceptions of Walking Distance to Neighbourhood Retail and Other Public Services. Transportation Research at McGill. School of Urban Planning, Faculty of Engineering at McGill University. Montreal, 2007.

HUBBERT, M. K. Energy From Fossil Fuels. Science, New Series, v. 109, n. 2823, p. 103-109, 1949.

HYDÉN, C.; NILSSON, A.; RISSER, R. How to Enhance Walking and Cycling Instead of Shorter Car Trips and to Make These Modes Safer. Department of Traffic Planning and Engineering, University of Lund, Sweden \& FACTUM Chaloupka, Praschl \& Risser OHG. Vienna, 1999.

LARSEN, J.; EL-GENEIDY, A.; YASMIN, F. Beyond the Quarter Mile: re-examining travel distances by active transportation. Canadian Journal of Urban Research: Canadian Planning and Policy, v. 19, n. 1, supplement, p. 70-88, 2010.

LOVELACE, R.; PHILIPS, I. The “Oil Vulnerability” of commuter patterns: a case study from Yorkshire and the Humber, UK. Geoforum, v. 51, p. 169-182, 2014.

MCCORMACK, G. R. et al. Objective Versus Perceived Walking Distances to Destinations. Environment and Behavior, v. 40, n. 3, 2008.

MOUDON, A. V. et al. Operational Definitions of Walkable Neighbourhood: theoretical and empirical insights. Journal of Physical Activity and Health, v. 3, n. 1, p. 99-117, 2006.

NEWMAN, P.; KENWORTHY, J. 'Peak Car Use': understanding the demise of automobile dependence. World Transport Policy and Practice, v. 17, n. 2, p. 31-42, 2011.

OLIVEIRA, A.; TAN, F.; RODRIGUES DA SILVA, A. Adequação do Modo de Transporte: um indicador de mobilidade sustentável em campus universitário. In: CONGRESSO

NACIONAL DE PESQUISA EM

TRANSPORTES DA ANPET, 30., Rio de Janeiro, Brasil, 2016. Anais... Rio de Janeiro, 2016. 
PHILIPS, I. The Potential Role of Walking and Cycling to Increase Resilience of Transport Systems to Future External Shocks. Leeds, 2014. (Thesis) - University of Leeds, Institute for Transport Studies, Leeds, 2014.

RENDALL, S. et al. Quantifying Transport Energy Resilience: active mode accessibility. Transportation Research Record: Journal of the Transportation Research Board, v. 2242, p. 72-80, 2011.

RODRIGUES DA SILVA, A. N.; COSTA, G. C. F.; BRONDINO, N. C. M. Urban Sprawl and Energy Use For Transportation in the Largest Brazilian Cities. Energy for Sustainable

Development, v. 11, n. 3, p. 44-50, 2007.

SAUNDERS, M. J.; RODRIGUES DA SILVA, A. N. Reducing Urban Transport Energy Dependence: a new urban development framework and GISbased tool. International Journal of Sustainable Transportation, v. 3, n. 2, p. 71-87, 2009.
SCHWANEN, T. Rethinking Resilience as Capacity to Endure. City, v. 20, n. 1, p. 152-160, 2016.

SHIM, G. E. et al. The Relationship Between the Characteristics of Transportation Energy Consumption and Urban Form. The Annals of Regional Science, v. 40, n. 2, p. 351-367, 2006.

WALKER, J.; COOPER, M. Genealogies of Resilience: from systems ecology to the political economy of crisis adaptation. Security Dialogue, v. 42, n. 2, p. 143-160, 2011.

YANG, Y.; DIEZ-ROUX, A. V. Walking Distance by Trip Purpose and Population Subgroups. American Journal of Preventive Medicine, v. 43, n. 1, p. 11-19, 2012.

ZHANG, W.; KOCKELMAN, K. M. Urban Sprawl, Job Decentralization, and Congestion: the welfare effects of congestion tolls and urban growth boundaries. In: TRANSPORTATION RESEARCH BOARD ANNUAL MEETING, 93., Washington, 2014. Proceedings... Washington, 2014.

\section{Marcel Carlos Martins}

Departamento de Engenharia de Transportes, Escola de Engenharia de São Carlos | Universidade de São Paulo | Av. Trabalhador Sãocarlense, 400, Centro | São Carlos - SP - Brasil | CEP 13566-590 | Tel.: (16) 3373-9595 | E-mail: mcmmartins91@gmail.com

Antônio Nélson Rodrigues da Silva

Departamento de Engenharia de Transportes, Escola de Engenharia de São Carlos | Universidade de São Paulo | E-mail: anelson@sc.usp.br

Revista Ambiente Construído

Associação Nacional de Tecnologia do Ambiente Construído

Av. Osvaldo Aranha, 99 - 3ำ andar, Centro

Porto Alegre - RS - Brasil

CEP $90035-190$

Telefone: +55 (51) 3308-4084

Fax: +55 (51) 3308-4054

www. seer. ufrgs. br/ ambienteconstruido

E-mail: ambienteconstruido@ufrgs.br 